\title{
Personalized treatment of hepatitis B
}

\author{
Anna S. Lok \\ Division of Gastroenterology and Hepatology, University of Michigan, Ann Arbor, MI, USA
}

There are seven approved drugs for treatment of hepatitis B. Professional guidelines provide a framework for managing patients but these guidelines should be interpreted in the context of the individual patient's clinical and social circumstances. Personalized management of hepatitis B can be applied based on prediction of the individual patient's risk of cirrhosis and hepatocellular carcinoma to guide the frequency and intensity of monitoring and urgency of treatment. It can also be applied to decisions regarding when to start treatment, which drug to use, and when to stop based on the individual patient's disease characteristics, preference, comorbidities and other mitigating circumstances. (Clin Mol Hepatol 2015;21:1-6)

Keywords: Hepatitis B virus; Chronic hepatitis B; Management; Personalized medicine

\section{INTRODUCTION}

Personalized medicine has gained a lot of attention in recent years with improved understanding of the contribution of genetic factors to benign and malignant diseases and responses to pharmacological interventions, and the availability of a wide spectrum of genetic tests at low costs. Although the term "personalized medicine" is often used to refer to individualizing care according to the genetic make-up of the patient, this term can be interpreted more broadly to mean "tailoring care to the individual patient characteristics". In other words, personalized medicine can be interpreted as an approach to classify individuals into subpopulations that differ in their susceptibility to a particular disease, rate of disease progression, and response to specific treatments such that preventative or therapeutic interventions can be concentrated on those who will benefit, sparing expense and side effects for those who will not. Simply put, the goal of personalized medicine is to provide optimal care according to the individual patient's dis- ease characteristics, personal preference, comorbidities, and social circumstances such that maximum benefit can be derived while minimizing costs and adverse reactions.

Substantial progress has been made in the treatment of hepatitis B in the last 15 years. There are currently seven approved drugs for the treatment of hepatitis $B$ : two formulations of interferon (IFN) - conventional and pegylated IFN (PEG-IFN), and five nucleos(t)ide analogues - lamivudine, telbivudine, adefovir, entecavir, and tenofovir. These drugs can suppress hepatitis B virus (HBV) replication, decrease hepatic inflammation and fibrosis and even reverse cirrhosis, prevent complications of cirrhosis, and reduce the incidence of hepatocellular carcinoma (HCC). ${ }^{1-3}$ However, currently approved drugs do not eradicate HBV and have low rates of clearance of hepatitis B e antigen ( $\mathrm{HBeAg}$ ) and hepatitis B surface antigen ( $\mathrm{HBsAg}$ ) and $\mathrm{HCC}$ continues to occur albeit at a lower rate. Because currently available treatment has little or no effect on covalently closed circular DNA, the template for transcription of HBV pregenomic RNA and messenger RNA and translation of viral

\section{Abbreviations:}

AASLD, American Association for the Study of Liver Diseases; ALT, alanine aminotransferase; APASL, Asian Pacific Association for the Study of the Liver, EASL, European Association for the Study of the Liver; $\mathrm{HBeAg}$, hepatitis B e antigen; $\mathrm{HBsAg}$, hepatitis B surface antigen; HBV, hepatitis B virus; HCC, hepatocellular carcinoma; IFN, interferon; PEG-IFN, pegylated interferon.

\section{Corresponding author : Anna S. Lok}

Division of Gastroenterology and Hepatology, University of Michigan, 3912 Taubman Center, SPC 5362, 1500 East Medical Center Drive, Ann Arbor, MI, USA

Tel: +1-734-936-7511, Fax: +1-734-936-7392

Email: aslok@umich.edu 
proteins or on restoration of host immune response to HBV, viral relapse occurs in most patients when treatment is stopped. Thus, most patients require many years and often lifelong treatment to derive continued benefit. Long durations of treatment are associated with increasing risks of adverse reactions, antiviral drug resistance, costs, and nonadherence to medications. Thus, a major dilemma in hepatitis $B$ treatment is when to initiate therapy. While all patients with chronic HBV infection are at risk of cirrhosis, liver failure and HCC, not all patients will experience these outcomes. Immune control of HBV with spontaneous loss of HBeAg and HBsAg can occur and patients can remain in remission for many years.

Professional society guidelines provide frameworks for managing patients with hepatitis B but these guidelines have to be interpreted in the context of the individual patient's clinical and social circumstances. Personalized management of hepatitis B can be applied based on prediction of the individual patient's risk of cirrhosis and HCC to guide the frequency and intensity of monitoring including HCC surveillance and urgency of treatment. It can also be applied to decisions on when to start treatment, which drug to use, and when to stop based on the individual patient's disease characteristics, preference, comorbidities and other mitigating circumstances.

\section{Prediction of risk of cirrhosis and HCC}

Host, viral and environmental factors contribute to an HBsAgpositive person's risk of cirrhosis and HCC (Table 1). Although several studies have identified genetic markers associated with increased risk of HBV-related HCC, these markers have not been validated in broad patient populations of diverse racial/ethnic origins infected with all HBV genotypes. Therefore, genetic markers have not yet been incorporated into prediction models for cirrhosis or HCC.

During the past decade, many models have been developed to predict the risk of cirrhosis or HCC in persons with chronic HBV infection. Some models incorporate many variables including blood test results such as HBV genotype, precore and core promoter variants that are not routinely available or clinical information such as alcohol and tobacco use that are unreliable unless the information is collected using standardized questionnaire. Table 2 summarizes three prediction models for HCC based on routinely available clinical and laboratory information: REACH-B, CU-HCC, and GAG$\mathrm{HCC}^{4-8}$ These models can be used to predict 5 - and 10 - year risk of HCC to guide the frequency of monitoring, the need for HCC surveillance, and the urgency of antiviral treatment. However, there are limitations to these models. These models were derived from cohorts of patients with chronic hepatitis B in Asia, who

Table 1. Factors associated with risks of hepatocellular carcinoma

1. Virus: HBV DNA level, HBV genotype, molecular variants (pre-S, basal core promoter, precore)

2. Host: sex, age, race/ethnicity, genetics, diabetes, cirrhosis

3. Environment: alcohol, cigarettes, coinfection with hepatitis C or D virus, aflatoxins

Table 2. Risk scores for predicting hepatocellular carcinoma

\begin{tabular}{|c|c|c|c|c|c|}
\hline & GAG-HCC ${ }^{8}$ & CUHK-HCC $^{5}$ & $\begin{array}{c}\text { REVEAL } \\
\text { Nomogram }^{\text {Original }}{ }^{6}\end{array}$ & $\begin{array}{c}\text { REVEAL } \\
\text { Nomogram } \\
\text { Updated }^{4}\end{array}$ & $\begin{array}{c}\text { REVEAL } \\
\text { Nomogram } \\
\text { REACH-B: } \\
\text { abbreviated }\end{array}$ \\
\hline \multicolumn{6}{|l|}{ Derivation Cohort } \\
\hline Geographical area & Hong Kong & Hong Kong & Taiwan & Taiwan & Taiwan \\
\hline Origin of subjects & Hospital based & Hospital based & Community based & Community based & Community based \\
\hline No. of subjects & 820 & 1005 & 2435 & 2435 & 3584 \\
\hline Risk predictors & $\begin{array}{l}\text { Gender } \\
\text { Age } \\
\text { HBV DNA level } \\
\text { Core promoter } \\
\text { mutations } \\
\text { Cirrhosis }\end{array}$ & $\begin{array}{l}\text { Age } \\
\text { Albumin } \\
\text { Bilirubin } \\
\text { HBV DNA level } \\
\text { Cirrhosis }\end{array}$ & $\begin{array}{l}\text { Gender } \\
\text { Age } \\
\text { HBeAg status } \\
\text { HBV DNA level } \\
\text { ALT level } \\
\text { HBV genotype } \\
\text { Alcohol consumption } \\
\text { Family history of HCC }\end{array}$ & $\begin{array}{l}\text { Gender } \\
\text { Age } \\
\text { HBeAg status } \\
\text { HBV DNA level } \\
\text { ALT level } \\
\text { HBV genotype } \\
\text { HBsAg level } \\
\text { Family history of HCC }\end{array}$ & $\begin{array}{l}\text { Gender } \\
\text { Age } \\
\text { HBeAg status } \\
\text { HBV DNA level } \\
\text { ALT level }\end{array}$ \\
\hline
\end{tabular}


were predominantly infected with HBV genotypes B and C and who did not receive antiviral treatment. Thus, these models may not be applicable in other parts of the world where the prevalent HBV genotypes are different and where HBV infection is mostly acquired in adult life and not early childhood or in patients receiving antiviral treatment. Indeed, two studies of patients receiving antiviral treatment in Europe found that performance of these scores, particularly the REACH-B score was poor. ${ }^{9,10}$ By contrast, one study in Hong Kong showed that all three models accurately predicted which patients with chronic hepatitis B treated with entecavir will develop HCC."

\section{When to initiate antiviral treatment?}

The American, Asian-Pacific and European Liver Association (AASLD, APASL and EASL) Guidelines recommend that treatment decision should be made based on clinical status, serum HBV DNA and alanine aminotransferase (ALT) levels, HBeAg status, and liver histology if available..$^{12-14}$ All guidelines recommend starting treatment as soon as possible in patients with life-threatening liver disease: acute liver failure, decompensated cirrhosis or severe acute exacerbation of chronic hepatitis B regardless of HBV DNA and ALT levels. These guidelines also recommend antiviral treatment for patients with compensated cirrhosis regardless of ALT levels but there are minor differences in threshold HBV DNA levels for initiating treatment. All guidelines agree that treatment should be initiated in noncirrhotic patients with serum HBV DNA levels greater than 20,000 IU/mL and persistently elevated ALT levels and/or histologic evidence of moderate or severe inflammation or fibrosis. However, cutoff values of HBV DNA and ALT and the need for liver biopsy or non-invasive assessment of liver fibrosis in determining treatment indications vary slightly among the guidelines. Because HBV DNA and ALT levels fluctuate during the course of chronic HBV infection, all guidelines agree that serial HBV DNA and ALT levels are more important than values at a single time point in making treatment decisions. Furthermore, all guidelines recommend that patients who are not started on treatment should be monitored such that treatment may be initiated at a later time when HBV replication or liver disease becomes more active.

The AASLD, APASL and EASL guidelines are based on a combination of scientific evidence and expert opinion and experience. These guidelines have not incorporated prediction models for cirrhosis or HCC into recommendations when or which patient should start treatment but incorporation of risk scores for HCC may be appropriate for Asian patients particularly those in the gray zone. The guidelines emphasize that patient age, family history of HCC, occupational requirements, plans to start a family (for women), and patient preference should also be considered in making treatment decisions. There are many settings when a patient's medical or social circumstances warrant a personalized approach that may be "at odds" with the guidelines but appropriate for that patient. For example, a 48 year old man who is HBeAg-positive with serial ALT 19-28 U/L and HBV DNA 3,000,000-400,000,000 $\mathrm{IU} / \mathrm{mL}$ with no evidence of cirrhosis would be considered to be in the immune tolerant phase and "not meet" criteria for treatment but starting this patient on treatment is appropriate because there is ample data showing that patients who remain HBeAg-positive after age 40 and those who have serum HBV DNA levels higher than 2,000 IU/mL after more than four decades of infection are at increased risk of cirrhosis and HCC. ${ }^{15,16}$ Treatment would also be appropriate in a 32 year old surgeon who has the same disease characteristics if suppression of viremia is a prerequisite for permission to continue his profession as a surgeon and to perform surgical operations (exposure-prone procedures). Treatment might also be appropriate in a 45 year old man who is $\mathrm{HBeAg}$ negative with ALT 25-35 U/L and HBV DNA 1,800-9,500 IU/mL if he has several family members with HCC. Other examples of personalized approach being "at odds" with guidelines may involve a decision to defer treatment when guidelines would recommend treatment. For example, a 35 year old woman who is HBeAg-negative with ALT 55-65 U/L and HBV DNA 28,000-75,000 IU/mL with no evidence of cirrhosis may choose to defer treatment if she wishes to start a family and is concerned about the safety of anti-HBV medications on fetal development. Another patient with the same disease characteristics may choose to defer treatment if she does not have insurance coverage for medications, or has contraindications to using interferon and is unwilling to commit to many years of nucleos(t)ide analogue therapy. Guidelines do cite these examples as situations where individualized decisions are appropriate.

\section{Which anti-HBV drug to use?}

Selection of the first-line anti-HBV drug should be based on the safety and efficacy of the drug, risk of drug resistance, cost of the drug, and patient preference. The main advantages of IFN include a finite duration of treatment and a higher rate of HBeAg and HBsAg loss but IFN has to be administered parenterally and is associated with a wide range of adverse reactions some of which can be serious. Nucleos(t)ide analogues are well tolerated but most pa- 
tients require many years or lifelong treatment. Entecavir, telbivudine and tenofovir have more potent antiviral activity, and entecavir and tenofovir have higher barriers to antiviral drug resistance.

All guidelines recommend initial treatment with PEG-IFN, entecavir or tenofovir as monotherapy. ${ }^{12-14}$ Because of cost concerns and the lack of access to tenofovir in some Asian countries when the most recent version of the APASL guidelines were released, lamivudine, adefovir or telbivudine were also recommended as firstline drugs in treatment-naïve patients. ${ }^{13}$ IFN is not recommended in patients with acute liver failure, decompensated cirrhosis or severe exacerbations of chronic hepatitis $B$ but it may be used with caution in patients with compensated cirrhosis.

While guidelines consider PEG-IFN, entecavir and tenofovir as equivalent first-line anti-HBV drugs, in clinical practice, entecavir and tenofovir are used much more often than PEG-IFN even in patients with no contraindications to the use of IFN. The decision to choose a nucleos(t)ide analogue over PEG-IFN may be based on the patient's preference for an oral drug with minimal adverse reactions over an injectable drug with many potential side effects but it may also be based on the physician's beliefs and bias. Drug cost is certainly an important factor in medical decisions in any society. Although cost-effectiveness studies have been performed, these analyses rely on many assumptions and decision on PEG-IFN versus nucleos(t)ide analogues is complicated because the effica$c y$, tolerability, and duration of these two classes of drugs are very different and the costs of these medications vary widely from one country to another.

The first step in deciding which drug to use for treating hepatitis $B$ is to choose between PEG-IFN versus nucleos(t)ide analogue. Patients' preference for route of administration (injection versus oral), duration of treatment (1 year versus many years), and presence of medical/psychiatric comorbidities that contraindicate use of or decrease tolerance to IFN are the most important deciding factors. A personalized decision on anti-HBV drug should also consider the likelihood of response. In this regard, the predictors of response to PEG-IFN and nucleos(t)ide analogues are similar: high ALT and low HBV DNA levels are the most reliable predictors of response to both PEG-IFN and nucleos(t)ide analogues. Thus, patients who are more likely to respond to PEG-IFN are also more likely to respond to nucleos(t)ide analogues. One predictor of response that is unique to PEG-IFN is HBV genotype although this applies mainly to HBeAg-positive patients. HBeAg-positive patients with genotype $A$ HBV have significantly higher rates of $\mathrm{HBeAg}$ and HBsAg loss compared to patients with genotypes $B, C$ or D..$^{17}$ Thus, HBeAg-positive patients with genotype A HBV and no contraindications to use of IFN should be encouraged to receive PEG-IFN. Interleukin-28B (IL28B) polymorphism was shown to be a strong predictor of response to IFN-based therapy for hepatitis $C$ but the association between IL28B polymorphism and PEG-IFN treatment of hepatitis B is inconsistent.

Other factors may play a role in personalized decisions regarding choice of anti-HBV drugs. For example, young patients may prefer a finite course of PEG-IFN versus many years of nucleos(t)ide analogue treatment. This is particularly true for young women contemplating to start a family in the next few years. Once a decision is made to treat with nucleos(t)ide analogues, the main considerations are cost and risk of antiviral drug resistance. If cost is not a concern, tenofovir and entecavir are preferred as these drugs have markedly lower rates of drug resistance (0-1\% after 5-6 years of continuous therapy) $)^{18,19}$ compared to lamivudine, adefovir or telbivudine. Tenofovir and entecavir have similary potency and barrier to antiviral resistance, and either drug may be used as first-line treatment in nucleoside-naive patients. Because of a low risk of nephrotoxicity, entecavir is preferred in older patients, patients with baseline impaired renal function and those with other medical conditions such as hypertension or diabetes that would increase the risk of renal insufficiency. Although telbivudine had been reported to improve renal function, ${ }^{20}$ the high risk of antiviral drug resistance and the potential for other adverse reactions such as myopathy and polyneuropathy make this a poor choice as firstline treatment. ${ }^{21}$ Tenofovir is effective in suppressing not only wild type HBV but also lamivudine, telbivudine or entecavir resistant $\mathrm{HBV}$ and to a slightly lesser extent adefovir resistant HBV; therefore, it is a better choice for patients who had been previously treated with other nucleos(t)ide analogues. Tenofovir is also a preferred choice for women of reproductive age because of its safety record in pregnancy. ${ }^{22}$

\section{When to stop treatment?}

AASLD, APASL and EASL guidelines recommend administration of PEG-IFN for 48-52 weeks in both HBeAg-positive and HBeAgnegative patients. ${ }^{12-14}$ There is some variation in recommendations when nucleos(t)ide analogues can be stopped. All guidelines recommend that in $\mathrm{HBeAg}$-positive patients, nucleos(t)ide analogues can be stopped when the patient has completed 6-12 months consolidation therapy after HBeAg seroconversion. The EASL guidelines recommend continuing treatment until HBsAg loss in patients with advanced fibrosis or cirrhosis to avoid flares associated with viral relapse. The AASLD and EASL guidelines recom- 
mend that in HBeAg-negative patients, nucleos(t)ide analogues should be continued until HBsAg loss but the APASL guidelines stated that treatment may be withdrawn after completing at least two years treatment with undetectable HBV DNA documented on three occasions six months apart. All three guidelines recommend lifelong nucleos(t)ide analogue treatment in patients with cirrhosis before treatment unless they had compensated cirrhosis and had cleared HBsAg.

Not all patients respond to PEG-IFN. In addition to pre-treatment factors, several studies have found decrease in HBsAg level after 12 or 24 weeks of PEG-IFN treatment to be better predictors of response. Specifically, a lack of or a small decline in HBsAg level after week 12 or 24 of PEG-IFN is associated with $85-95 \%$ negative predictive value of a response. ${ }^{23}$ These data suggest that a response-guided stop-rule may be used making PEG-IFN therapy more attractive because patients who are unlikely to benefit can be spared from further side effects and costs. A recent cost-effective study showed that response-guided PEG-IFN therapy is the most cost-effective approach for HBeAg-positive but not HBeAgnegative patients. ${ }^{24}$ Thus, HBeAg-positive patients with no contraindications, particularly those with genotype A HBV, high ALT and low HBV DNA levels before treatment who are already predicted to have a high likelihood of response to PEG-IFN may be further encouraged to have a trial of PEG-IFN, treatment can be stopped after 12 weeks if decline in HBsAg is inadequate. A caveat to this approach is that it has not been validated prospectively and different stop rules have been proposed by different authors and for different HBV genotypes.

For patients receiving nucleos(t)ide analogue therapy, the likelihood of achieving HBeAg seroconversion is $40-50 \%$ and the likelihood of achieving HBsAg loss is $0-10 \%$ after 5 years of continuous treatment. ${ }^{3}$ Thus, more than half of the HBeAg-positive patients and $\geq 95 \%$ of HBeAg-negative patients will require more than 5 years of treatment. A few studies found that while viral relapse (redetection of HBV DNA in serum) occurs in all HBeAg-negative patients who completed 2-5 years treatment and who stopped treatment before HBsAg loss, clinical relapse with elevated ALT and HBV DNA levels $>2,000 \mathrm{IU} / \mathrm{mL}$ occurred in only $50 \%$ of patients. ${ }^{25,26}$ These data suggest that HBeAg-negative patients who have completed $>2$ years treatment may consider stopping treatment if they are unwilling to continue or unable to afford continued treatment. However, this personalized approach must be accompanied by a plan for close monitoring for at least 6 months after discontinuation of treatment such that treatment can be promptly resumed if necessary.

\section{CONCLUSIONS}

Personalized approach to treatment of hepatitis B should adapt practice guidelines to individual patient's disease characteristics, personal preferences, medical comorbidities and social circumstances to determine when treatment should be started, which drug to use, and when to stop treatment, such that maximum

Table 3. Personalized treatment of hepatitis B

-When to start treatment

- Predicted risk of disease progression, cirrhosis and HCC

- Assessment of HBV replication status and activity / stage of liver disease

- Other considerations: age, medical comorbidities, plans to start a family (for women), occupational requirements (for health care workers engaged in exposure prone procedures), health insurance coverage, willingness to commit to injection therapy or long durations of oral therapy, perception of seriousness of liver disease and benefits/risks of treatment

- Which drug to use

- Liver disease: any cirrhosis, portal hypertension, severe hepatitis flare or liver failure

- Medical or psychiatric contraindications to use of IFN

- Likelihood of response: ALT and HBV DNA level, HBV genotype (for IFN)

- Patient preference: acceptance of injection versus oral therapy, adverse reactions, duration of treatment

-When to stop treatment

- IFN: finite duration or response-guided therapy for HBeAg-positive patients

- Nucleos(t)ide analogues: indefinite treatment vs. trial of stopping treatment

- HBeAg-positive: after HBeAg seroconversion and 12 months consolidation therapy

- HBeAg-negative: after 3-5 years treatment with persistently undetectable HBV DNA 
benefit can be derived while minimizing costs and adverse reactions (Table 3).

\section{Conflicts of Interest}

The author has no conflicts to disclose.

\section{REFERENCES}

1. Liaw YF, Sung JJ, Chow WC, Farrell G, Lee CZ, Yuen H, et al. Lamivudine for patients with chronic hepatitis $B$ and advanced liver disease. N Engl J Med 2004;351:1521-1531.

2. Marcellin P, Gane E, Buti M, Afdhal N, Sievert W, Jacobson IM, et al. Regression of cirrhosis during treatment with tenofovir disoproxil fumarate for chronic hepatitis B: a 5-year open-label follow-up study. Lancet 2013;381:468-475.

3. Yapali S, Talaat N, Lok AS. Management of hepatitis B: our practice and how it relates to the guidelines. Clin Gastroenterol Hepatol 2014;12:16-26.

4. Lee $\mathrm{MH}$, Yang HI, Liu J, Batrla-Utermann R, Jen CL, Iloeje UH, et al. Prediction models of long-term cirrhosis and hepatocellular carcinoma risk in chronic hepatitis B patients: risk scores integrating host and virus profiles. Hepatology 2013;58:546-554.

5. Wong VW, Chan SL, Mo F, Chan TC, Loong HH, Wong GL, et al. Clinical scoring system to predict hepatocellular carcinoma in chronic hepatitis B carriers. J Clin Oncol 2010;28:1660-1665.

6. Yang HI, Sherman M, Su J, Chen PJ, Liaw YF, Iloeje UH, et al. Nomograms for risk of hepatocellular carcinoma in patients with chronic hepatitis B virus infection. J Clin Oncol 2010;28:2437-2344.

7. Yang HI, Yuen MF, Chan HL, Han KH, Chen PJ, Kim DY, et al. Risk estimation for hepatocellular carcinoma in chronic hepatitis B (REACHB): development and validation of a predictive score. Lancet Oncol 2011;12:568-574.

8. Yuen MF, Tanaka Y, Fong DY, Fung J, Wong DK, Yuen JC, et al. Independent risk factors and predictive score for the development of hepatocelIular carcinoma in chronic hepatitis B. J Hepatol 2009;50:80-88.

9. Arends P, Sonneveld MJ, Zoutendijk R, Carey I, Brown A, Fasano M, et al. Entecavir treatment does not eliminate the risk of hepatocellular carcinoma in chronic hepatitis B: limited role for risk scores in Caucasians. Gut 2014; doi:10.1136/gutjnl-2014-307023.

10. Vlachogiannakos J, Papatheodoridis GV. Optimal therapy of chronic hepatitis B: how do I treat HBeAg-positive patients? Liver Int 2015;35(Suppl 1):100-106.

11. Wong GL, Chan HL, Chan HY, Tse PC, Tse YK, Mak CW, et al. Accuracy of risk scores for patients with chronic hepatitis $B$ receiving entecavir treatment. Gastroenterology 2013;144:933-944.

12. EASL. EASL Clinical Practice Guidelines: Management of chronic hepatitis B virus infection. J Hepatol 2012;57:167-185.

13. Liaw YF, Kao JH, Piratvisuth T, Chan H, Chien RN, Liu CJ, et al. Asian-Pacific consensus statement on the management of chronic hepatitis B: a 2012 update. Hepatol Int 2012;6:531-561.

14. Lok AS, McMahon BJ. Chronic hepatitis B: update 2009. Hepatology 2009;50:661-662.

15. Chu CM, Liaw YF. Chronic hepatitis B virus infection acquired in childhood: special emphasis on prognostic and therapeutic implication of delayed HBeAg seroconversion. J Viral Hepat 2007;14:147152.

16. Chen CJ, Yang HI, Su J, Jen CL, You SL, Lu SN, et al. Risk of hepatocellular carcinoma across a biological gradient of serum hepatitis $B$ virus DNA level. JAMA 2006;295:65-73.

17. Buster EH, Hansen BE, Lau GK, Piratvisuth T, Zeuzem S, Steyerberg EW, et al. Factors that predict response of patients with hepatitis B e antigen-positive chronic hepatitis B to peginterferon-alfa. Gastroenterology 2009;137:2002-2009.

18. Kitrinos KM, Corsa A, Liu Y, Flaherty J, Snow-Lampart A, Marcellin P, et al. No detectable resistance to tenofovir disoproxil fumarate after 6 years of therapy in patients with chronic hepatitis B. Hepatology 2014;59:434-442.

19. Tenney DJ, Rose RE, Baldick CJ, Pokornowski KA, Eggers BJ, Fang $J$, et al. Long-term monitoring shows hepatitis $B$ virus resistance to entecavir in nucleoside-naive patients is rare through 5 years of therapy. Hepatology 2009;49:1503-1514.

20. Gane EJ, Deray G, Liaw YF, Lim SG, Lai CL, Rasenack J, et al. Telbivudine improves renal function in patients with chronic hepatitis $B$. Gastroenterology 2014;146:138-146.

21. Yapali S, Lok AS. Potential benefit of telbivudine on renal function does not outweigh its high rate of antiviral drug resistance and other adverse effects. Gastroenterology 2014;146:15-19.

22. Antiretoviral pregnancy registry, interim report, 1/1/1989-7/31/2014. http://www.apregistry.com/forms/interim_report.pdf. Accessed 3/9/15.

23. Konerman MA, Lok AS. Is It More Cost-effective for Patients With Chronic Hepatitis B to Have a Trial of Interferon Before Considering Nucleos(t)ide Analogue Therapy? Clin Gastroenterol Hepatol 2015;13;386-389.

24. Lo AO, Wong VW, Wong GL, Chan HL, Dan YY. Cost Effectiveness of Response-Guided Therapy With Peginterferon in the Treatment of Chronic Hepatitis B. Clin Gastroenterol Hepatol 2015;13:377-385.e5.

25. Hadziyannis SJ, Sevastianos V, Rapti I, Vassilopoulos D, Hadziyannis E. Sustained responses and loss of HBsAg in HBeAg-negative patients with chronic hepatitis $B$ who stop long-term treatment with adefovir. Gastroenterology 2012;143:629-636.e1.

26. Jeng WJ, Sheen IS, Chen YC, Hsu CW, Chien RN, Chu CM, et al. Off-therapy durability of response to entecavir therapy in hepatitis B e antigen-negative chronic hepatitis B patients. Hepatology 2013;58:1888-1896 